\title{
TOPOLOGICAL ERGODIC THEORY AND MEAN ROTATION
}

\author{
STEVE ALPERN AND V. S. PRASAD
}

(Communicated by Charles C. Pugh)

Dedicated to the memory of John C. Oxtoby (1910-1991)

whose influence has been a driving force in our work

\begin{abstract}
Let $\mathscr{L}_{0}\left(M^{n}, \mu\right)$ denote the set of all homeomorphisms of a compact manifold $M^{n}$ that preserve a locally positive nonatomic Borel probability measure $\mu$ and are isotopic to the identity. The notion of the mean rotation vector for a torus homeomorphism has been extended by Fathi to a continuous map $\theta$ on $\mathscr{H}_{0}\left(M^{n}, \mu\right)$. We show that any abstract ergodic behavior typical for automorphisms of $\left(M^{n}, \mu\right)$ as a Lebesgue space is also typical not only in $\mathscr{H}_{0}\left(M^{n}, \mu\right)$ but also in each closed subset of constant $\theta$. By typical we mean dense $G_{\delta}$ in the appropriate space. Weak mixing is an example of such a typical abstract ergodic behavior. This contrasts sharply with a deep result of the KAM theory that for some rotation vectors $\vec{v}$, there is an open neighborhood of rotation by $\vec{v}$, in the space of smooth volume preserving $n$-torus diffeomorphisms with $\theta=\vec{v}$, where each diffeomorphism in the open set is conjugate to rotation by $\vec{v}$ (and hence cannot be weak mixing).
\end{abstract}

\section{INTRODUCTION}

For an orientation preserving area preserving homeomorphism of the torus, the mean rotation vector $\theta(h)$ measures the fractional part of the mean translation of any planar lift of $h$. Fathi [Fa] has extended this notion by defining $\theta$ continuously on the space $\mathscr{H}_{0}\left(M^{n}, \mu\right)$ of all homeomorphisms of a compact connected manifold $M^{n} \quad(n \geq 2)$ that preserve a locally positive nonatomic Borel probability measure $\mu$ and are isotopic to the identity. Roughly speaking, Fathi's definition of $\theta(h)$ measures the net twisting action of $h$ in each of the nontrivial circle directions. The aim of this paper is to examine the relation, if any, between a mean rotation vector $\vec{v}$ and the typical (dense $G_{\delta}$ in the topology of uniform convergence) ergodic properties of homeomorphisms in $\mathscr{H}^{\vec{v}}\left(M^{n}, \mu\right) \equiv \theta^{-1}(\vec{v})$.

An earlier result of Alpern [A12] is that any measure theoretic property that is typical (dense $G_{\delta}$ ) in the weak topology for the group of invertible measure preserving transformations is also typical for $\mathscr{H}\left(M^{n}, \mu\right)$, the set of

Received by the editors August 19, 1991; the results of this paper were presented at the conference on Symbolic Dynamics and its Applications, held at Yale University, New Haven, CT, July 28-August 2, 1991.

1991 Mathematics Subject Classification. Primary 58F11; Secondary 28D05.

The authors gratefully acknowledge support from a NATO Collaborative research grant. 
$\mu$-preserving homeomorphisms with the topology of uniform convergence. This paper strengthens Alpern's result by showing that every typical measure theoretic property is typical not only in $\mathscr{H}_{0}\left(M^{n}, \mu\right)$, but also in each section $\mathscr{H}^{\vec{v}}\left(M^{n}, \mu\right)$ with mean rotation vector $\vec{v}$. In particular, weak mixing is such a property and our main theorem establishes the existence of weak mixing homeomorphisms with prescribed mean rotation vectors. This application of our main theorem extends the result of Katok and Stepin [KS] that weak mixing homeomorphisms are dense $G_{\delta}$ in $\mathscr{H}\left(M^{n}, \mu\right)$. Our theorem also extends the classical result of Oxtoby and Ulam [OU] that ergodic homeomorphisms are typical in $\mathscr{H}\left(M^{n}, \mu\right)$.

An application of our theorem is to show that the deep results of the Kolmogorov-Arnold-Moser (KAM) theory (see for example [Mo] or [He]) do not extend to the continuous category. The KAM theorem asserts that if $\vec{v} \in R^{n}$ is a vector satisfying a certain diophantine condition, then any sufficiently small perturbation of $R^{\vec{v}}$, the rotation on $T^{n}$ by $\vec{v}$, by a smooth volume preserving rotationless (i.e., has $\theta=\overrightarrow{0}$ ) diffeomorphism still remains conjugate to rotation $R^{\vec{v}}$. However, our main theorem shows that any $R^{\vec{v}}$ can be composed with arbitrarily small rotationless volume preserving homeomorphisms to produce any typical measure theoretic behavior; in particular, we can get properties like weak mixing that exclude these perturbations from being rotation (since weak mixing transformations have no rotation factors (see $[\mathrm{Ru}])$ ).

A further application of our results is to give yet another proof of the Poincaré-Birkhoff theorem on fixed points of area preserving twist maps of the annulus. Any area preserving homeomorphism of the annulus that twists the bounding circles in opposite directions can be extended to an area preserving homeomorphism of a larger annulus, which has no new fixed points and has mean rotation zero. Our main result implies that this extension can be approximated by an ergodic mean rotation zero homeomorphism. It can be shown, using results of Atkinson [At] and Schmidt [Sc], that the lift to the strip $I \times R$ of any ergodic mean rotation zero homeomorphism of the annulus is recurrent. Hence, following Franks [Fr], the Brouwer Plane Translation Theorem implies that the lift, and hence the original map, has a fixed point. The details are carried out in $[\mathrm{AP}]$.

\section{ROTATION VECTORS AND PRELIMINARIES}

Let $M^{n}$ be a compact connected $n$-dimensional manifold, $\mu$ a nonatomic Borel probability measure that is zero on boundary and positive on open sets, and $\mathscr{K}_{0}\left(M^{n}, \mu\right)$ the group of $\mu$ preserving homeomorphisms of $M^{n}$ onto itself isotopic to the identity.

For a $\mu$ preserving homeomorphism $h$ of the torus $T^{n}$, the rotation vector of $h$ is defined to be the vector in $R^{n}$ whose $i$ th coordinate is

$$
\int_{[0,1)^{n}}\left(p_{i}(\hat{h}(x))-p_{i}(x)\right) \mu(d x)
$$

where $\hat{h}$ is a lift of $h$ to the covering space $R^{n}$ and $p_{i}$ is projection onto the $i$ th coordinate. Two lifts of the same homeomorphism will have rotation vectors that differ by integer multiples in each coordinate direction. Consequently, we identify these vectors as the rotation vector of $h$. 
For homeomorphisms of manifolds other than the torus we need to identify the nontrivial circle directions on the manifold and then measure the action of the homeomorphism on these directions. This is what Fathi did in [Fa] using his mass flow homomorphism.

Theorem 1 [Fa]. There is a continuous group homomorphism

$$
\theta: \mathscr{H}_{0}\left(M^{n}, \mu\right) \rightarrow H_{1}\left(M^{n}, R\right) / \Gamma
$$

called the mass flow homomorphism, where $H_{1}\left(M^{n}, R\right)$ is the first homology group and $\Gamma$ is some discrete subgroup of $H_{1}\left(M^{n}, R\right)$.

Consequently, for each $\vec{v} \in H_{1}\left(M^{n}, R\right)$ let

$$
\mathscr{H}^{\vec{v}}\left(M^{n}, \mu\right)=\left\{h \in \mathscr{H}_{0}\left(M^{n}, \mu\right): \theta(h)=\vec{v}\right\} .
$$

Then $\mathscr{H}^{\vec{v}}\left(M^{n}, \mu\right)$ is a closed (and hence Baire) subspace of $\mathscr{H}_{0}\left(M^{n}, \mu\right)$ with the topology of uniform convergence.

For the reader's convenience, the following description of the mass flow homomorphism is taken from Fathi's paper. First define a continuous homomorphism $\tilde{\theta}$ from the space of paths of homeomorphisms $\widetilde{\mathscr{H}}_{0}\left(M^{n}, \mu\right)$ to $H_{1}\left(M^{n}, R\right)$ the first homology group of $M^{n}$. Observe that $H_{1}\left(M^{n}, R\right)=$ $\operatorname{Hom}\left(\left[M^{n}, T^{1}\right], R\right)$, where $\left[M^{n}, T^{1}\right]$ is the set of homotopy classes of maps from $M^{n}$ to $T^{1}=R / Z$. Note that the group law on the circle $T^{1}$ is written additively. Given $\left(h_{t}\right) \in \widetilde{\mathscr{H}}_{0}\left(M^{n}, \mu\right)$, the homomorphism $\tilde{\theta}\left(h_{t}\right):\left[M^{n}, T^{1}\right] \rightarrow R$ is defined as follows:

Let $p: M^{n} \rightarrow T^{1}$ be continuous. Because $p h_{t}-p: M^{n} \rightarrow T^{1}$ is a homotopy that satisfies $p h_{0}-p=0$, we can lift it to a homotopy $\overline{p h_{t}-p}: M^{n} \rightarrow R$ such that $\overline{p h_{0}-p}=0$. Fathi defines

$$
\tilde{\theta}\left(h_{t}\right)(p)=\int_{M^{n}} \overline{p h_{1}-p} d \mu .
$$

This yields a homomorphism $\left[M^{n}, T^{1}\right] \rightarrow R$ that depends only on the homotopy class of $\left(h_{t}\right)$; furthermore, $\tilde{\theta}: \widetilde{\mathscr{H}}_{0}\left(M^{n}, \mu\right) \rightarrow H_{1}\left(M^{n}, R\right)$ is a group homomorphism. The covering projection $\widetilde{\mathscr{H}}_{0}\left(M^{n}, \mu\right) \rightarrow \mathscr{H}_{0}\left(M^{n}, \mu\right)$ is a group homomorphism having the fundamental group of $\mathscr{H}_{0}\left(M^{n}, \mu\right)$ as kernel. Let $\Gamma$ be the image under $\tilde{\theta}$ of this kernel. By passing to the quotient we get the group homomorphism $\theta: \mathscr{H}_{0}\left(M^{n}, \mu\right) \rightarrow H_{1}\left(M^{n}, R\right) / \Gamma$, which is called the mass flow homomorphism. If $\operatorname{Hom}\left(\left[M^{n}, T^{1}\right], R\right)$ has the weak topology, then $\tilde{\theta}$ (and, therefore, $\theta)$ is continuous; furthermore, $\theta$ is surjective if $H_{1}\left(M^{n}, R\right)$ has a basis represented by embedded curves having tubular neighborhoods.

As an illustration of the mass flow homomorphism, Fathi gives the following

Example. Let $M^{n}=T^{n}$, the $n$-Torus, and $\mu$ be Haar measure. By taking the $n$ projections $p_{i}: T^{n} \rightarrow T^{1},\left(x_{1}, \ldots, x_{n}\right) \mapsto x_{i}$ as a basis (over $Z$ ) of $\left[M^{n}, T^{1}\right]$, then $\operatorname{Hom}\left(\left[M^{n}, T^{1}\right], R\right)$ can be identified with $R^{n}$. Let $\vec{v} \in R^{n}$ and let the isotopy $R_{t}^{\vec{v}}(x)=x+t \vec{v}$; a simple calculation shows that $\tilde{\theta}\left(R_{t}^{\vec{v}}\right)=$ $\vec{v}$. The discrete subgroup $\Gamma=Z^{n}$. Furthermore, for any homomorphism $h \in \mathscr{H}_{0}\left(M^{n}, \mu\right)$ the numbers $\tilde{\theta}(h)\left(p_{i}\right)$ give the same numbers as the rotation vector defined at the beginning of the section. 
Here are some other facts that we will need.

We consider $\mathscr{H}_{0}\left(M^{n}, \mu\right) \subset \mathscr{G}\left(M^{n}, \mu\right)$, the group of invertible, measurable, and measure preserving transformations of $\left(M^{n}, \mu\right)$ onto itself. On $\mathscr{G}\left(M^{n}, \mu\right)$ we define two complete metrizable topologies with metrics for $\mathscr{G}\left(M^{n}, \mu\right)$ (where $f, g \in \mathscr{G}\left(M^{n}, \mu\right)$ and $d$ is the metric on $\left.M^{n}\right)$ :

(1) The norm topology: $d_{n}(f, g)=\left\|f g^{-1}\right\|=\operatorname{ess} \sup d(f(x), g(x))$.

(2) The weak topology: $d_{w}(f, g)=\inf \{\delta: \mu\{x: d(f(x), g(x)) \geq \delta\}<$ $\delta\}$.

The weak topology (which should properly be called the topology of convergence in measure the way we have defined it) is discussed by Halmos using a different definition in [Ha] - see [A11] for a proof of the equivalence of these two definitions. The norm topology on $\mathscr{G}\left(M^{n}, \mu\right)$ has the property that its restriction to $\mathscr{H}_{0}\left(M^{n}, \mu\right)$ yields the topology of uniform convergence. It is clear that the norm topology is stronger than the weak topology.

Measure theoretically, all Lebesgue spaces are the same. Topologically, $M^{n}$ can be obtained from the $n$-dimensional cube $I^{n}$ by making boundary identifications. These two observations can be combined to give

Proposition 1. For $M^{n}$ a compact connected n-manifold and $\mu$ a good Borel measure on $M^{n}$, there is a continuous map $\phi: I^{n} \rightarrow M^{n}$ such that

(1) $\phi$ is surjective;

(2) $\phi \mid$ Int $I^{n}$ is a homeomorphism onto its image;

(3) $\phi\left(\partial I^{n}\right) \cap \phi\left(\operatorname{Int} I^{n}\right)=\varnothing$ and $\phi\left(\partial I^{n}\right)$ is a closed nowhere dense set of $\mu$ measure 0 ;

(4) $\lambda \phi^{-1}(E)=\mu(E)$, for all Borel $E \subset M^{n}$, where $\lambda$ is Lebesgue measure on $I^{n}$.

Proof. See [KS, OU, Fa] for complete details. We outline the proof: Morton Brown proved (1), (2), and the fact that $\phi\left(\partial I^{n}\right)$ is closed, nowhere dense, and disjoint from $\phi\left(\mathrm{Int} I^{n}\right)$ (see $[\mathrm{Br}]$ or [CV]). Then since any closed nowhere dense set can be homeomorphed to one of measure zero, we can choose $\phi$ so that $\phi\left(\partial I^{n}\right)$ has measure zero. An application of the homeomorphic measures theorem of von Neumann, Oxtoby and Ulam [OU] shows that $\phi$ can be chosen so that (4) holds.

The next proposition is Alpern's extension of Halmos's conjugacy lemma [Ha] to the topological setting. A proof of the proposition uses Proposition 1 to reduce this to the case when $\left(M^{n}, \mu\right)=\left(I^{n}, \lambda\right)$ and can be found in [Al2].

Proposition 2 (Topological Conjugacy Approximation). Given $\varepsilon>0$ and $h$, $g_{1} \in \mathscr{G}\left(M^{n}, \mu\right)$, where $g_{1}$ is antiperiodic (i.e., $\mu\left\{x: g_{1}^{n}(x)=x\right\}=0$ for all $n)$, there is a conjugate $g=f g_{1} f^{-1}, f \in \mathscr{G}\left(M^{n}, \mu\right)$, of $g_{1}$ so that $d(g(x), h(x))<\varepsilon$ for $\mu$-a.e. $x \in M^{n}$.

In the following proposition the Norm Bounded Density property (NBD) for a manifold (see [Al2]), which states that a sufficiently small invertible measurable transformation can be weakly approximated by a small homeomorphism, is modified so that the approximating homeomorphism has null rotation vector.

Proposition 3 (Lusin Approximation). For each $\varepsilon>0$, there is $a \delta>0$, so that given $r \in \mathscr{G}\left(M^{n}, \mu\right)$ with $d(r(x), x)<\delta, \mu$-a.e., and a weak neighborhood $V$ 
or $r$, there is a homeomorphism $f \in \mathscr{L}_{0}\left(M^{n}, \mu\right)$ so that $f \in V, d(f(x), x)<$ $\varepsilon$, and $\theta(f)=\overrightarrow{0}$.

Proof. For $\phi$ the map from Proposition 1, let $0<\varepsilon_{1}<\omega_{\phi}(\varepsilon)$ and choose $0<\delta<\omega_{\phi^{-1}}\left(\varepsilon_{1}\right)$, where $\omega_{\phi}$ and $\omega_{\phi^{-1}}$ are the moduli of continuity of $\phi$ and $\phi^{-1}$, respectively. Since $\phi\left(\partial I^{n}\right)$ is a $\mu$-null set, $\hat{r}=\phi^{-1} r \phi \in \mathscr{G}\left(I^{n}, \lambda\right)$. By our choice of $\delta$, we see that $d(\hat{r}(y), y)<\varepsilon_{1}$ a.e. $y \in I^{n}$. Apply the Lusin Theorem in [Al1] to $\hat{r} \in \mathscr{G}\left(I^{n}, \lambda\right)$ to find $\hat{f} \in \mathscr{H}\left(I^{n}, \lambda\right)$ with $\hat{f} \in \phi^{-1} V \phi$, a weak neighborhood of $\hat{r} ; d(\hat{f}(y), y)<\varepsilon_{1}$ a.e.; and $\hat{f} \mid \partial I^{n}=\mathrm{id}$.

Then $f=\phi \hat{f} \phi^{-1}$ is our required homeomorphism. To see this, first note that $f \in V$ and $d(f(x), x)<\varepsilon$ a.e. $x$ by choice of $\varepsilon_{1}$. Let $B=M^{n} \backslash \phi\left(\partial I^{n}\right)$ and observe that $f \mid \phi\left(\partial I^{n}\right)=$ id. Since $\hat{f}$ is isotopic to the identity, by conjugating with $\phi$, we can choose $\left(f_{t}\right)$ as an isotopy of $f$ to the identity with the property that $f_{t} \mid \phi\left(\partial I^{n}\right)=$ id. To calculate the rotation vector of $f$ we use Fathi's proof of his Lemma $5.3[\mathrm{Fa}]$. Let $p: M^{n} \rightarrow T^{1}$ be a continuous map. Then since $p f_{t}-p$ vanishes outside of $B$ so does the lift $\overline{p f_{t}-p}: M^{n} \rightarrow R$. Therefore,

$$
\tilde{\theta}\left(f_{t}\right)(p)=\int_{B} \overline{p f_{1}-p} d \mu .
$$

Since $B$ is homeomorphic to the contractible space $\operatorname{Int}\left(I^{n}\right), p \mid B: B \rightarrow T^{1}$ can be lifted to a map $\bar{p}: B \rightarrow R$ (see [CV, Chapter 13]). Using the uniqueness of the lift and the fact that $f_{t}(B)=B$, we get $\overline{p f_{t}-p}\left|B=\bar{p} f_{t}\right| B-\bar{p}$. Since $f_{1}$ is $\mu$ preserving, we get $\tilde{\theta}\left(f_{t}\right)(p)=\int_{B} \overline{p f_{1}-p} d \mu=\int_{B}\left(\bar{p} f_{1} \mid B-\bar{p}\right) d \mu=0$.

\section{MAIN THEOREM}

We can now state our main theorem.

Theorem 2. Let $\mathscr{P} \subset \mathscr{G}\left(M^{n}, \mu\right)$ be a conjugate invariant set (i.e., $g \mathscr{P} g^{-1} \subset \mathscr{P}$ for all $\left.g \in \mathscr{G}\left(M^{n}, \mu\right)\right)$ that is a dense $G_{\delta}$ subset of $\mathscr{G}\left(M^{n}, \mu\right)$ in the weak topology. Choose $\vec{v} \in H_{1}\left(M^{n}, R\right)$ for which $\mathscr{H}^{\vec{v}}\left(M^{n}, \mu\right)$ is nonempty. Then $\mathscr{P} \cap \mathscr{C}^{\vec{v}}\left(M^{n}, \mu\right)$ is a dense $G_{\delta}$ subset of $\mathscr{C}^{\vec{v}}\left(M^{n}, \mu\right)$ with the topology of uniform convergence.

Proof. Let $h \in \mathscr{H}^{\vec{v}}\left(M^{n}, \mu\right) \subset \mathscr{G}\left(M^{n}, \mu\right)$ and $\varepsilon>0$ be given. Let $\mathscr{P}=$ $\bigcap_{k=1}^{\infty} \mathscr{P}_{k}$ be a representation of $\mathscr{P}$ as a weak dense $G_{\delta}$ subset of $\mathscr{G}\left(M^{n}, \mu\right)$. Each $\mathscr{P}_{k}$ therefore, is open in the norm topology in $\mathscr{G}\left(M^{n}, \mu\right)$. Let $U(h, \varepsilon)=$ $\left\{f \in \mathscr{G}\left(M^{n}, \mu\right): d(f(x), h(x))<\varepsilon, \mu\right.$-a.e. $\}$ be a norm ball around $h \in$ $\mathscr{G}\left(M^{n}, \mu\right)$. Using Baire's theorem, we only need to show

$$
\mathscr{P}_{k} \cap U(h, \varepsilon) \cap \mathscr{H}^{\vec{v}}\left(M^{n}, \mu\right) \neq \varnothing
$$

where $\mathscr{P}_{k}$ is a weak dense open set. Since $\mathscr{P}$ is a weak dense $G_{\delta}$ subset of $\mathscr{G}\left(M^{n}, \mu\right)$, there is a $g_{1} \in \mathscr{P} \cap \mathscr{E}$ (where $\mathscr{E}$ is the dense $G_{\delta}$ subset of $\mathscr{G}\left(M^{n}, \mu\right)$ consisting of ergodic transformations) so that $g_{1}$ is antiperiodic. Now apply the conjugacy result of Proposition 2 to $h, g_{1}$ and use the fact that $\mathscr{P}$ is conjugate invariant to get $g \in \mathscr{P} \cap U\left(h, \delta_{0}\right)$, where $\delta_{0}<\min (\delta, \varepsilon / 2)$. Then apply Proposition 3 to $r=g h^{-1}$ and the weak neighborhood $\mathscr{P}_{k} h^{-1}$ of $r$ to get an $f \in \mathscr{H}_{0}\left(M^{n}, \mu\right)$ with $f \in \mathscr{P}_{k} h^{-1}, d\left(f(x), g h^{-1}(x)\right)<\varepsilon / 2 \mu$-a.e., and $\theta(f)=\overrightarrow{0}$. Then $f h \in \mathscr{P}_{k} \cap U(h, \varepsilon) \cap \mathscr{H}^{\vec{v}}\left(M^{n}, \mu\right)$. 
Corollary 1. The weak mixing homeomorphisms are dense $G_{\delta}$ not only in $\mathscr{H}\left(M^{n}, \mu\right)$ but also in each $\mathscr{H}^{\vec{v}}\left(M^{n}, \mu\right)$ and hence in $\mathscr{H}_{0}\left(M^{n}, \mu\right)$ as well.

\section{REFERENCES}

[Al1] S. Alpern, Approximation to and by measure preserving homeomorphisms, J. London Math. Soc. (2) 18 (1978), 305-315.

[A12] _ Generic properties of measure preserving homeomorphisms, Ergodic Theory, Lecture Notes in Math., vol. 729, Springer, Berlin and New York, 1979, pp. 16-27.

[AP] S. Alpern and V. S. Prasad, Typical recurrence for lifts of mean rotation zero annulus homeomorphisms, Bull. London Math. Soc. 23 (1991), 477-481.

[At] G. Atkinson, Recurrence of co-cycles and random walks, J. London Math. Soc. (2) 13 (1976), 486-488.

[Br] M. Brown, A mapping theorem for untriangulated manifolds, Topology of 3-Manifolds and Related Results (M. K. Fort, ed.), Prentice-Hall, Englewood Cliffs, NJ, 1963, pp. 92-94.

[CV] C. Christenson and W. Voxman, Aspects of topology, Marcel Dekker, New York, 1977.

[Fa] A. Fathi, Structure of the group of measure preserving homeomorphisms preserving a good measure on a compact manifold, Ann. Sci. École Norm. Sup. (4) 13 (1980), 45-93.

[Fr] J. Franks, Recurrence and fixed points of surface homeomorphisms, Ergodic Theory and Dynamical Systems 8 (1988), 99-107.

[Ha] P. Halmos, Lectures on ergodic theory, Publ. Math. Soc. Japan, Tokyo, 1956; reprint, Chelsea, New York, 1960.

[He] M. R. Herman, Sur la conjugaison différentiable des difféomorphismes du cercle à des rotations, Inst. Hautes Études Sci. Publ. Math. 49 (1979), 1-233.

[KS] A. Katok and A. Stepin, Metric properties of measure preserving homeomorphisms, Uspekhi Mat. Nauk. 25 (1970), 193-220; English transl., Russian Math. Surveys 25, 191-220.

[Mo] J. Moser, A rapidly convergent iteration method, Part II, Ann. Scuola Norm. Sup. Pisa Cl. Sci. (3) 20 (1966), 499-535.

[OU] J. C. Oxtoby and S. Ulam, Measure preserving homeomorphisms and metrical transitivity, Ann. of Math. (2) 42 (1941), 874-920.

[Ru] D. J. Rudolph, Fundamentals of measurable dynamics. Ergodic theory on Lebesgue spaces, Oxford Univ. Press, London, 1990.

[Sc] K. Schmidt, Cocycles of ergodic transformation groups, Macmillan Lectures in Math., Macmillan, New Delhi, 1977.

Department of Mathematics, London School of Economics, Houghton Street, LONDON WC2A 2AE, UNITED KINGDON

E-mail address: alpern@vax2.lse.ac.uk

Department of Mathematics, University of Massachusetts at Lowell, Lowell, MASSACHUSETTS 01854

E-mail address: prasadv@woods.ulowell.edu 